\title{
Wave Structures for Nonlinear Schrodinger Types Fractional Partial Differential Equations Arise in Physical Sciences
}

\author{
Mst. Nasrin Nahar, Md. Tarikul Islam*, Diganta Broto Kar
}

Department of Mathematics, Hajee Mohammad Danesh Science and Technology University, Dinajpur, BANGLADESH

${ }^{*}$ Corresponding Contact:

Email: tarikulhstu1980@gmail.com

\begin{abstract}
Nonlinear partial differential equations are mostly renowned for depicting underlying behavior of nonlinear phenomena relating to the nature of real world. In this paper, we discuss for analytic solutions of fractional order nonlinear Schrodinger types equations such as the space-time fractional nonlinear Schrodinger equation and the (2+1)-dimensional time-fractional Schrodinger equation. The considered equations are converted into ordinary differential equations with the help of wave variable transformation and then the recently established rational $\left(D_{\xi}^{\alpha} G / G^{2}\right)$-expansion method is employed to construct the exact solutions. The obtained solutions are appeared in the forms of trigonometric function, hyperbolic function and rational function which are compared with those of literature and claimed to be different. The graphical representations of the solutions are finally brought out for their physical appearances. The applied method is seemed to be efficient, concise and productive which might be used for further research.
\end{abstract}

Mathematics Subject Classifications: 35C08, 35R11

Key words:

Rational (D_ $\left.\xi^{\wedge} \alpha G / G^{\wedge} 2\right)$-expansion method, Wave variable transformation, Nonlinear fractional partial differential equation, Analytic solution, Soliton

\section{INTRODUCTION}

In many fields of science, it is important to integrate and solve nonlinear partial derivative differential equations that contain derivative by time. The results of fractional nonlinear wave models have presented an advanced success in applied mathematics and engineering, mathematical physics, soliton physics. These fractional nonlinear wave models become much devotion in several other areas like signal processing, psychology, finance, acoustics, 
biology systems, medical processes, chemical physics, biology, fluid mechanics, medical process, and many more (Laskin, 2002; Eslami et al., 2004). Recently, the search for a different type of solution of the fractional nonlinear Schrodinger's model has represented numerous scientists and researchers (Saxena \& Kalla, 2010; Abdel-Salam et al., 2016; Younis et al., 2017; Rizvi et al., 2017). The established methods available in the literature are $G^{\prime} / G$ expansion method for nonlinear fractional differential equations by Bekir and Guner (2013), the meshless method of lines (Mohyud-Din, 2012), Adomian decomposition scheme (Guo, 2019), the new generalized $G^{\prime} / G$-expansion method (Alam et al., 2014; Alam, 2015; Alam \& $\mathrm{Li}, 2019)$, the Darcy law method (Sheikholeslami, 2017), the reproducing kernel algorithm (Omar, 2019a), topological solitons for certain differential equation by Biswas et al. (2013a), Biswas et al. (2013b), Laplace-Adomian decomposition method (Shah et al., 2019), reproducing kernel Hilbert space method (Omar, 2019b), homotopy perturbation method (Golmankhaneh \& Baleanu, 2011), improved sub-equation method (Karaagac, 2019), Schrodinger's equation (Rizvi et al., 2017; Li et al., 2019), generalized exponential rational function method (Ghanbari et al., 2019), the Kudryashov methods (Saha, 2016; Kudryashov, 2012), integral transform based decomposition methods are used for Schrodinger and other differential equations by Nuruddeen (2017); Nuruddeen \& Nass (2017), Various phenomena such as shallow water waves and multicellular biology dynamics arising in the nonlinear physical science (Lu et al., 2017; Bazyar \& Song, 2017), the $\left(G^{\prime} / G, 1 / G\right)$-expansion method (Zayed \& Abdelaziz, 2012; Zayed et al., 2018; Zayed \& Alurrfi, 2016), the Jacobi collocation method (Doha et al., 2014; Bhrawy et al., 2014a; Bhrawy et al., 2014b)], the improved tanh method (Islam \& Akter, 2021a), the rational $\left(G^{\prime} / G\right)$-expansion method (Jannah et al., 2021) etc. In lower the summary of this paper shown. In the $2^{\text {nd }}$ section, conformable fractional derivative and methodology are displayed, in the $3^{\text {rd }}$ section application of this method, in the $4^{\text {th }}$ section graphical representations are shown, the $5^{\text {th }}$ section is for conclusion.

\section{Preliminaries and Methodology}

\section{Conformable fractional derivative}

Let $\omega:[0, \infty) \rightarrow \boldsymbol{R}$ be a function. The $\alpha^{\prime} s$ order conformable derivative of $u$ is defined by Khalil et al. (2014)

$D_{x}^{\alpha}(\omega(x))=\lim _{h \rightarrow 0} \frac{\omega\left(x+h x^{1-\alpha}\right)-\omega(x)}{h}$

for all $x>0$ and $\alpha \epsilon(0,1)$. Further, the following theorems gives some properties of conformable derivative:

Theorem 1. Let $\alpha \epsilon(0,1)$ and suppose $\omega(x)$ and $\varrho(x)$ are $\alpha$-differentiable at $x>0$. Then

(i). $D_{x}^{\alpha}\left(x^{\varsigma}\right)=\varsigma x^{\varsigma-\alpha}$, for all $\varsigma \in \boldsymbol{R}$.

(ii). $D_{x}^{\alpha}(\beta)=0, \beta$ for all constant function $\omega(x)=\beta$.

(iii). $D_{x}^{\alpha}(\beta \omega(x))=\beta D_{x}^{\alpha} \omega(x)$, for all $\beta$ constant.

(iv). $D_{x}^{\alpha}(\beta \omega(x)+\gamma \varrho(x))=\beta D_{x}^{\alpha} \omega(x)+\gamma D_{x}^{\alpha} \varrho(x)$, for all $\beta, \gamma \in R$.

(v). $D_{x}^{\alpha}(\varrho(x) \omega(x))=\varrho(x) D_{x}^{\alpha}(\omega(x))+\omega(x) D_{x}^{\alpha}(\varrho(x))$.

(vi). $D_{x}^{\alpha}\left(\frac{\omega(x)}{\varrho(x)}\right)=\frac{\varrho(x) D_{x}^{\alpha} \omega(x)-\omega(x) D_{x}^{\alpha} \varrho(x)}{\varrho^{2}(x)}, \varrho \neq 0$.

(vii). If, in addition to $\omega(x)$ differentiable, then $D_{x}^{\alpha} \omega(x)=x^{1-\alpha} \frac{d \omega}{d x}$. 
Theorem 2. Let $\alpha \epsilon(0,1)$ such $\omega(x)$ is differentiable and also $\alpha$-differentiable. Let $\varrho(x)$ be a function defined in the range of $\omega(x)$ also differentiable, then

$D_{x}^{\alpha}(\omega(x) \cdot \varrho(x))=x^{1-\alpha} \varrho^{\prime}(x) \omega^{\prime}(\varrho(x))$.

\section{Methodology}

In this section, we introduce the newly established rational $\left(D_{\xi}^{\alpha} G / G^{2}\right)$-expansion method (Islam \& Akter, 2021b) for finding exact analytic solutions of nonlinear partial differential equations. Consider the FNLEE in the independent variables $t, x_{1}, x_{2}, \ldots, x_{n}$ as

$\mathrm{Q}\left(u_{1}, \ldots, u_{k}, D_{t}^{\alpha} u_{1}, \ldots, D_{t}^{\alpha} u_{k}, D_{x_{1}}^{\beta} u_{1}, \ldots, D_{x_{1}}^{\beta} u_{k}, \ldots D_{x_{n}}^{\beta} u_{1}, \ldots D_{x_{n}}^{\beta} u_{k}, \ldots\right)=0$,

where $u_{i}=u_{i}\left(t, x_{1}, x_{2}, \ldots, x_{n}\right), i=1, \ldots, k$ are unknown functions, $F$ is a polynomial in $u_{i}$ and it's various partial derivatives of fractional order.

Making use of the fractional composite transformation

$u_{i}=u_{i}\left(t, x_{1}, x_{2}, \ldots, x_{n}\right)=U_{i}(\xi), \xi=\xi\left(t, x_{1}, x_{2}, \ldots, x_{n}\right)$

Eq. (2.2.1) is turned into the following ordinary differential equation of fractional order with respect to the variable $\xi$ :

$Q\left(u_{1}, \ldots, u_{k}, D_{\xi}^{\alpha} u_{1}, \ldots, D_{\xi}^{\alpha} u_{k}, D_{\xi}^{\beta} u_{1}, \ldots, D_{\xi}^{\beta} u_{k}, \ldots\right)=0$

We may, if possible, take the anti-derivative of Eq. (2.2.3) term by term one or more times and integral constant can be set to zero as soliton solutions are sought.

Now, we discuss the main steps of the above-mentioned method to investigate exact analytic solutions of FNLEEs as follows:

First step: Suppose the solution to be in the form

$U(\xi)=\frac{\sum_{i=0}^{n} \iota_{i}\left(D_{\xi}^{\alpha} G / G^{2}\right)^{i}}{\sum_{i=0}^{n} \tau_{i}\left(D_{\xi}^{\alpha} G / G^{2}\right)^{i}}$

where at least one of $\iota_{n}$ and $\tau_{n}$ is nonzero; $\iota_{i}(i=0,1,2, \ldots, n), \tau_{i}(i=0,1,2, \ldots, n)$ and $d$ are unknown parameters, and $G=G(\xi)$ satisfies the following auxiliary nonlinear ordinary differential equation of fractional order:

$G^{2} D_{\xi}^{2 \alpha} G-(2 G+\lambda)\left(D_{\xi}^{\alpha} G\right)^{2}-\mu G^{4}=0$

It can be written as

$D_{\xi}^{\alpha}\left(D_{\xi}^{\alpha} G / G^{2}\right)=\mu+\lambda\left(D_{\xi}^{\alpha} G / G^{2}\right)^{2}$

The nonlinear fractional composite wave variable transformation $G(\xi)=H(\eta), \eta=\xi^{\alpha} / \alpha$ reduces Eq. (2.2.5) into the following ODE:

$H^{2} H^{\prime \prime}-(2 G+\lambda) H^{2}-\mu H^{4}=0$

whose solutions are well-known. Since $D_{\xi}^{\alpha} G(\xi)=D_{\xi}^{\alpha} H(\eta)=H^{\prime}(\eta) D_{\xi}^{\alpha} \eta=H^{\prime}(\eta)$, with the aid of the solutions of Eq. (2.2.7), we can obtain the solutions of Eq. (2.2.5) as follows:

$$
\begin{array}{ll}
\left(D_{\xi}^{\alpha} G / G^{2}\right)=\sqrt{\mu / \lambda} \times \frac{A \cos \sqrt{\lambda \mu} \xi^{\alpha} / \alpha+B \sin \sqrt{\lambda \mu} \xi^{\alpha} / \alpha}{B \cos \sqrt{\lambda \mu} \xi^{\alpha} / \alpha-A \sin \sqrt{\lambda \mu} \xi^{\alpha} / \alpha^{\prime}} & \lambda \mu>0 \\
\left(D_{\xi}^{\alpha} G / G^{2}\right)=-\sqrt{|\lambda \mu| / \lambda} \times \frac{A \sinh \left(2 \sqrt{\lambda \mu} \xi^{\alpha} / \alpha\right)+A \cosh \left(2 \sqrt{\lambda \mu} \xi^{\alpha} / \alpha\right)+B}{A \sinh \left(2 \sqrt{\lambda \mu} \xi^{\alpha} / \alpha\right)+A \cosh \left(2 \sqrt{\lambda \mu} \xi^{\alpha} / \alpha\right)-B^{\prime}} & \lambda \mu<0
\end{array}
$$




$$
\left(D_{\xi}^{\alpha} G / G^{2}\right)=-\frac{A \alpha}{\lambda\left(A \xi^{\alpha}+B \alpha\right)^{\prime}} \quad \mu=0, \lambda \neq 0
$$

Second step: Due to the theme of homogenous balance, Eq. (2.2.3) serves the value of $n$ appeared in Eq. (2.2.4).

Third step: Use Eqs. (2.2.4) and (2.2.5) in Eq. (2.2.3) with the value of $n$ found in second step to obtain a polynomial in $\left(D_{\xi}^{\alpha} G / G^{2}\right)$. Set each coefficient of this polynomial to zero and solve them by computer software Maple to gain the values of the unknown parameters available in Eq. (2.2.4).

Fourth step: Utilizing the values calculated at third step in Eq. (2.2.4) along with Eqs. (2.2.8)(2.2.10) makes available exact analytic solutions to Eq. (2.2.1).

\section{Application Of the Method}

The well-known space and time fractional nonlinear Schrodinger equation is

$i D_{t}^{\alpha} u+D_{x}^{2 \beta} u+2|u|^{2} u=0, t>0,0<\alpha, \beta \leq 1$,

where $x$ is the spatial variable and $t$ represents time. This equation occurs in non-linear optics, superconductivity and plasma physics. We introduce the fractional composite transformation as follows:

$u(x, t)=e^{i \varphi} u(\xi), \varphi=m^{1 / \beta} x+k^{1 / \alpha} t, \xi=x+r^{1 / \alpha} t$,

where $r, k$ and $m$ are arbitrary constants to be determined later. Eq. (3.1.1) with the aid of Eq. (3.1.2) reduces to one whose real part and imaginary part are given as:

$-\left(k+m^{2}\right) u+D_{\xi}^{2 \alpha} u+2 u^{3}=0$,

$r D_{\xi}^{\alpha} u+2 m D_{\xi}^{\alpha} u=0$,

where $u$ and its various derivatives are functions of $\xi$. Eq. (3.1.4) yields $=-\frac{r}{2}$, so that Eq. (3.1.3) becomes

$-\left(4 k+r^{2}\right) u+8 u^{3}+4 D_{\xi}^{2 \alpha} u=0$.

Balancing the terms $D_{\xi}^{2 \alpha} u$ and $u^{3}$ provides $n=1$ and the solution Eq. (2.2.4) takes the form

$u(\xi)=\frac{\iota_{0}+\iota_{1}\left(D_{\xi}^{\alpha} G(\xi) / G^{2}(\xi)\right)}{\tau_{0}+\tau_{1}\left(D_{\xi}^{\alpha} G(\xi) / G^{2}(\xi)\right)}$.

Inserting Eq. (3.1.6) with its essential derivatives and Eq. (2.2.6) into Eq. (3.1.5) makes a polynomial in $\left(D_{\xi}^{\alpha} G(\xi) / G^{2}(\xi)\right)$. Collect each coefficient of this polynomial and set to zero, we get a system of algebraic equations for $\iota_{0}, \iota_{1}, \tau_{0}, \tau_{1}, r$ and $k$ whose values are obtained by solving these equations by computational software Maple as follows:

Set $1: \iota_{0}:= \pm i \mu \tau_{1} ; \iota_{1}:=0 ; \tau_{0}:=0 ; k:=-\frac{r^{2}}{4}+2 \mu \lambda$;

Set $2: \iota_{0}:=\mp i \mu \tau_{1} ; \iota_{1}:= \pm i \tau_{0} \lambda ; k:=-\frac{r^{2}}{4}+2 \mu \lambda$;

Inserting the values from Eq. (3.1.7) and Eq. (3.1.8) into Eq. (3.1.6) yields

$u_{1}(\xi)=\frac{ \pm i \mu e^{i \varphi}}{\left(D_{\xi}^{\alpha} G / G^{2}\right)^{\prime}}$ 
$u_{2}(\xi)=e^{i \varphi} \times \frac{\mp i \mu \tau_{1} \pm i \tau_{0} \lambda\left(D_{\xi}^{\alpha} G / G^{2}\right)}{\tau_{0}+\tau_{1}\left(D_{\xi}^{\alpha} G / G^{2}\right)}$,

Eqs. (3.1.9), (3.1.10) together with the Eqs. (2.2.8)-(2.2.10) make available the following outcomes:

$u_{1}^{1,2}(\xi)= \pm e^{i \varphi} \sqrt{\frac{\lambda}{\mu}} \times \frac{i \mu \times\left(B \cos \sqrt{\lambda \mu} \xi^{\alpha} / \alpha-A \sin \sqrt{\lambda \mu} \xi^{\alpha} / \alpha\right)}{A \cos \sqrt{\lambda \mu} \xi^{\alpha} / \alpha+B \sin \sqrt{\lambda \mu} \xi^{\alpha} / \alpha}, \lambda \mu>0$,

where $\varphi=(-r / 2)^{1 / \alpha} x+\left(\left(8 \lambda \mu-r^{2}\right) / 4\right)^{1 / \alpha} t, \xi=x+r^{1 / \alpha} t$.

$u_{1}^{3,4}(\xi)=\mp e^{i \varphi} \sqrt{\frac{\lambda}{|\lambda \mu|}} \times \frac{i \mu \times\left\{A \sinh \left(2 \sqrt{\lambda \mu} \xi^{\alpha} / \alpha\right)+A \cosh \left(2 \sqrt{\lambda \mu} \xi^{\alpha} / \alpha\right)-B\right\}}{A \sinh \left(2 \sqrt{\lambda \mu} \xi^{\alpha} / \alpha\right)+A \cosh \left(2 \sqrt{\lambda \mu} \xi^{\alpha} / \alpha\right)+B}, \lambda \mu<0$,

where $\varphi=(-r / 2)^{1 / \alpha} x+\left(\left(8 \lambda \mu-r^{2}\right) / 4\right)^{1 / \alpha} t, \xi=x+r^{1 / \alpha} t$.

$u_{2}^{5,6}(\xi)=e^{i \varphi} \times \frac{\mp i \mu \tau_{1} \pm i \tau_{0} \sqrt{\lambda \mu} \times \frac{A \cos \sqrt{\lambda \mu} \xi^{\alpha} / \alpha+B \sin \sqrt{\lambda \mu} \xi^{\alpha} / \alpha}{B \cos \sqrt{\bar{\lambda} \mu} \xi^{\alpha} / \alpha-A \sin \sqrt{\lambda \mu} \xi^{\alpha} / \alpha}}{\tau_{0}+\tau_{1} \sqrt{\mu / \lambda} \times \frac{A \cos \sqrt{\lambda \mu} \xi^{\alpha} / \alpha+B \sin \sqrt{\lambda \mu} \xi^{\alpha} / \alpha}{B \cos \sqrt{\lambda \mu} \xi^{\alpha} / \alpha-A \sin \sqrt{\lambda \mu} \xi^{\alpha / \alpha}}}, \lambda \mu>0$,

where $\varphi=(-r / 2)^{1 / \alpha} x+\left(\left(8 \lambda \mu-r^{2}\right) / 4\right)^{1 / \alpha} t, \xi=x+r^{1 / \alpha} t$.

$u_{2}^{7,8}(\xi)=e^{i \varphi} \times \frac{\mp i \mu \tau_{1} \mp i \tau_{0} \sqrt{\lambda|\lambda \mu|} \times \frac{A \sinh \left(2 \sqrt{\lambda \mu} \xi^{\alpha} / \alpha\right)+A \cosh \left(2 \sqrt{\lambda \mu} \xi^{\alpha} / \alpha\right)+B}{A \sinh \left(2 \sqrt{\lambda \mu} \xi^{\alpha} / \alpha\right)+A \cosh \left(2 \sqrt{\lambda \mu} \xi^{\alpha} / \alpha\right)-B}}{\tau_{0}-\tau_{1} \sqrt{|\lambda \mu| / \lambda} \times \frac{A \sinh \left(2 \sqrt{\lambda \mu} \xi^{\alpha} / \alpha\right)+A \cosh \left(2 \sqrt{\lambda \mu} \xi^{\alpha} / \alpha\right)+B}{A \sinh \left(2 \sqrt{\lambda \mu} \xi^{\alpha} / \alpha\right)+A \cosh \left(2 \sqrt{\lambda \mu} \xi^{\alpha} / \alpha\right)-B}}, \lambda \mu<0$,

where $\varphi=(-r / 2)^{1 / \alpha} x+\left(\left(8 \lambda \mu-r^{2}\right) / 4\right)^{1 / \alpha} t, \xi=x+r^{1 / \alpha} t$.

$u_{2}^{9,10}(\xi)=e^{i \varphi} \times \frac{\mp i \tau_{0} \times \frac{A \alpha}{\left(A \xi^{\alpha}+B \alpha\right)}}{\tau_{0}-\tau_{1} \times \frac{A \alpha}{\lambda\left(A \xi^{\alpha}+B \alpha\right)}}, \mu=0, \lambda \neq 0$,

where $\varphi=(-r / 2)^{1 / \alpha} x+\left(\left(8 \lambda \mu-r^{2}\right) / 4\right)^{1 / \alpha} t, \xi=x+r^{1 / \alpha} t$.

The above obtained solutions are compared with those of (Kaplan et al., 2016) and seemed to be different which first time recorded in the literature.

3.2. The (2+1)-dimensional time-fractional Schrodinger equation

The (2+1)-dimensional time-fractional Schrodinger equation is

$i D_{t}^{\alpha} u+\rho u_{x x}-\eta u_{y y}+\tau u|u|^{2}=0$,

where $\rho, \eta$ and $\tau$ are unknown parameters.

The consideration

$u(x, y, t)=u(\xi), \xi=x+y+r^{1 / \alpha} t$,

forces Eq. (3.2.1) to become

$\operatorname{ir} D_{\xi}^{\alpha} u+\rho D_{\xi}^{2 \alpha} u-\eta D_{\xi}^{2 \alpha} u+\tau u|u|^{2}=0$.

with

$u(\xi)=e^{i k \xi} v(\xi)$,

where $v(\xi)$ is a real-valued function and $k$ is unknown parameter. Equating real and imaginary parts from Eq. (3.2.3) yields 
$r D_{\xi}^{\alpha} v+2 k(\rho-\eta) D_{\xi}^{\alpha} v=0$

$(\rho-\eta) D_{\xi}^{2 \alpha} v+\tau v^{3}-\left(k r+k^{2}(\rho-\eta)\right) v=0$.

From Eq. (3.2.5),

$$
k=\frac{r}{2(\eta-\rho)} \text {. }
$$

Eq. (3.2.6) reduces to

$4(\rho-\eta)^{2} D_{\xi}^{2 \alpha} v+4 \tau(\rho-\eta) v^{3}+r^{2} v=0$.

Due to the homogenous balance principle, we attain $n=1$ and solution (2.2.4) turns into

$v(\xi)=\frac{\iota_{0}+\iota_{1}\left(D_{\xi}^{\alpha} G(\xi) / G^{2}(\xi)\right)}{\tau_{0}+\tau_{1}\left(D_{\xi}^{\alpha} G(\xi) / G^{2}(\xi)\right)}$.

Eq. (3.2.8) with solution (3.2.9) and Eq. (2.2.5) becomes a polynomial in $\left(D_{\xi}^{\alpha} G(\xi) / G^{2}(\xi)\right)$. Set each coefficient to zero and solve by computational software Maple for the following outcomes:

Set 1: $\iota_{0}:=0 ; \iota_{1}:= \pm \sqrt{\frac{2(\eta-\rho)}{\sigma}} \tau_{0} \lambda ; \tau_{1}=0 ; r= \pm 2 \sqrt{-2 \lambda \mu}(\eta-\rho)$;

Set 2: $\iota_{0}:= \pm \sqrt{\frac{2(\eta-\rho)}{\sigma}} \tau_{1} \mu ; \iota_{1}:=\mp \sqrt{\frac{2(\eta-\rho)}{\sigma}} \tau_{0} \lambda ; r= \pm 2 \sqrt{-2 \lambda \mu}(\eta-\rho) ;$

Operating the values available in Eqs. (3.2.10)-(3.2.11) into Eq. (3.2.9) yields the following expressions for chosen solutions:

$u_{1}(\xi)= \pm e^{i k \xi} \times \sqrt{\frac{2(\eta-\rho)}{\sigma}} \lambda\left(D_{\xi}^{\alpha} G / G^{2}\right)$,

where $= \pm \sqrt{-2 \lambda \mu}, \xi=x+y+( \pm 2 \sqrt{-2 \lambda \mu}(\eta-\rho))^{1 / \alpha} t$.

$u_{2}(\xi)=e^{i k \xi} \times \frac{\sqrt{\frac{2(\eta-\rho)}{\sigma}}\left\{ \pm \tau_{1} \mu \mp \tau_{0} \lambda\left(D_{\xi}^{\alpha} G / G^{2}\right)\right\}}{\tau_{0}+\tau_{1}\left(D_{\xi}^{\alpha} G / G^{2}\right)}$,

where $= \pm \sqrt{-2 \lambda \mu}, \xi=x+y+( \pm 2 \sqrt{-2 \lambda \mu}(\eta-\rho))^{1 / \alpha} t$.

Eq. (3.2.13) and Eq. (3.2.14) along with Eqs. (2.2.8)-(2.2.10) provide the following exact solutions:

$u_{1}^{1,2}(\xi)= \pm e^{i k \xi} \sqrt{\frac{2 \mu \lambda(\eta-\rho)}{\sigma}} \times \frac{A \cos \sqrt{\lambda \mu} \xi^{\alpha} / \alpha+B \sin \sqrt{\lambda \mu} \xi^{\alpha} / \alpha}{B \cos \sqrt{\lambda \mu} \xi^{\alpha} / \alpha-A \sin \sqrt{\lambda \mu} \xi^{\alpha} / \alpha}, \lambda \mu>0$,

where $= \pm \sqrt{-2 \lambda \mu}, \xi=x+y+( \pm 2 \sqrt{-2 \lambda \mu}(\eta-\rho))^{1 / \alpha} t$.

$u_{1}^{3,4}(\xi)=\mp e^{i k \xi} \sqrt{\frac{2 \lambda|\lambda \mu|(\eta-\rho)}{\sigma}} \times \frac{A \sinh \left(2 \sqrt{\lambda \mu} \xi^{\alpha} / \alpha\right)+A \cosh \left(2 \sqrt{\lambda \mu} \xi^{\alpha} / \alpha\right)+B}{A \sinh \left(2 \sqrt{\lambda \mu} \xi^{\alpha} / \alpha\right)+A \cosh \left(2 \sqrt{\lambda \mu} \xi^{\alpha} / \alpha\right)-B}, \lambda \mu<0$,

where $= \pm \sqrt{-2 \lambda \mu}, \xi=x+y+( \pm 2 \sqrt{-2 \lambda \mu}(\eta-\rho))^{1 / \alpha} t$.

$u_{1}^{5,6}(\xi)=\mp e^{i k \xi} \sqrt{\frac{2(\eta-\rho)}{\sigma}} \times \frac{A \alpha}{A \xi^{\alpha}+B \alpha}, \mu=0, \lambda \neq 0$, 
where $= \pm \sqrt{-2 \lambda \mu}, \xi=x+y+( \pm 2 \sqrt{-2 \lambda \mu}(\eta-\rho))^{1 / \alpha} t$

$u_{2}^{7,8}(\xi)=e^{i k \xi} \times \frac{\sqrt{\frac{2(\eta-\rho)}{\sigma}}\left\{ \pm \tau_{1} \mu \mp \tau_{0} \sqrt{\lambda \mu} \times \frac{A \cos \sqrt{\lambda \mu} \xi^{\alpha} / \alpha+B \sin \sqrt{\lambda \mu} \xi^{\alpha} / \alpha}{B \cos \sqrt{\lambda \mu} \xi^{\alpha} / \alpha-A \sin \sqrt{\lambda \mu} \xi^{\alpha} / \alpha}\right\}}{\tau_{0}+\tau_{1} \sqrt{\mu / \lambda} \times \frac{A \cos \sqrt{\lambda \mu} \xi^{\alpha} / \alpha+B \sin \sqrt{\lambda \mu} \xi^{\alpha} / \alpha}{B \cos \sqrt{\lambda \mu} \xi^{\alpha} / \alpha-A \sin \sqrt{\lambda \mu} \xi^{\alpha} / \alpha}}, \lambda \mu>0$,

where $= \pm \sqrt{-2 \lambda \mu}, \xi=x+y+( \pm 2 \sqrt{-2 \lambda \mu}(\eta-\rho))^{1 / \alpha} t$.

$u_{2}^{2}(\xi)=e^{i k \xi} \times \frac{\sqrt{\frac{2(\eta-\rho)}{\sigma}}\left\{ \pm \tau_{1} \mu \pm \tau_{0} \sqrt{\lambda|\lambda \mu|} \times \frac{A \sinh \left(2 \sqrt{\lambda \mu} \xi^{\alpha} / \alpha\right)+A \cosh \left(2 \sqrt{\lambda \mu} \xi^{\alpha} / \alpha\right)+B}{A \sinh \left(2 \sqrt{\lambda \mu} \xi^{\alpha} / \alpha\right)+A \cosh \left(2 \sqrt{\lambda \mu} \xi^{\alpha} / \alpha\right)-B}\right\}}{\tau_{0}-\tau_{1} \sqrt{|\lambda \mu| / \lambda} \times \frac{A \sinh \left(2 \sqrt{\lambda \mu} \xi^{\alpha} / \alpha\right)+A \cosh \left(2 \sqrt{\lambda \mu} \xi^{\alpha} / \alpha\right)+B}{A \sinh \left(2 \sqrt{\lambda \mu} \xi^{\alpha} / \alpha\right)+A \cosh \left(2 \sqrt{\lambda \mu} \xi^{\alpha} / \alpha\right)-B}}, \lambda \mu<0$,

where $= \pm \sqrt{-2 \lambda \mu}, \xi=x+y+( \pm 2 \sqrt{-2 \lambda \mu}(\eta-\rho))^{1 / \alpha} t$.

The above achieved solutions are compared with the existing results in the literature which shown the novelty of our results (Rizvi et al., 2017).

\section{Graphical Representations of the Obtained Solutions}

The solutions furnished above to the suggested equations are brought out graphically for their physical appearances. The outlined solitons take different shape such as kink, bell, cuspon, peakon, periodic etc. For simplicity, we display few graphs as follows:

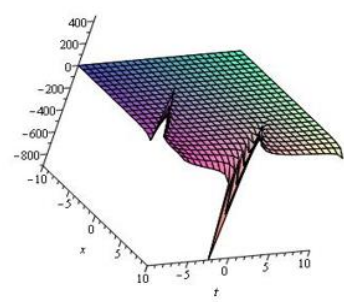

Figure 1: The plot of solution (3.1.11) $\quad$ for $\alpha=1, \beta=1, r=$ $1, \lambda=3, \mu=.02, \mathrm{~m}=.5, \mathrm{~A}=$ $3, B=1$ within $-10 \leq x, t \leq 10$.

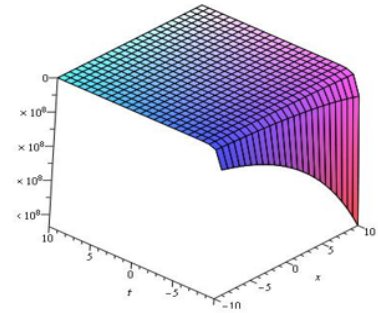

Figure 4: The shape of solution (3.1.14) $\quad$ for $\alpha=1, \beta=1, r=$ $3, \lambda=0.2, \mu=-.05, \tau_{0}=$ $0.2, \tau_{1}=0.1, \mathrm{~m}=5, \mathrm{~A}=4, B=$ 1 within $-10 \leq x, t \leq 10$.

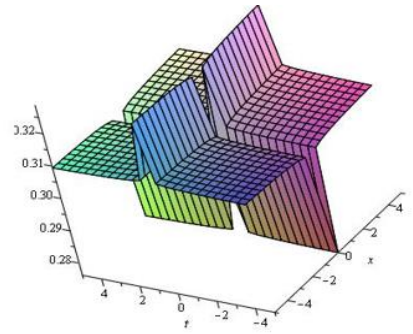

Figure 2: The outline of solution (3.1.12) for $\alpha=.001, \mu=-.02$,

$\beta=.001, r=.002, \lambda=.04, \quad \mathrm{~m}=$ $.003, \mathrm{~A}=3, B=1$ within $-5 \leq$ $x, t \leq 5$.

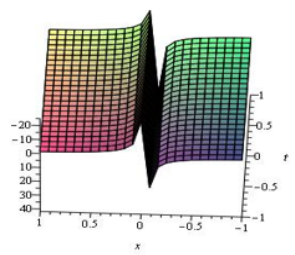

Figure 5: The singular king shape soliton of solution (3.1.15) for $\alpha=1, \beta=1, r=.01, \lambda=$ $0.02, \tau_{0}=0.05, \tau_{1}=0.01, \mathrm{~m}=$ $0.3, \mathrm{~A}=4, B=1$ within $\quad-1 \leq$ $x, t \leq 1$.

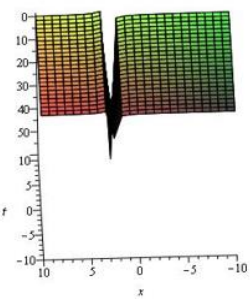

Figure 3: The shape of solution (3.1.13) for $\alpha=\beta=B=1, \lambda=$ $2, r=0.003, \tau_{1}=0.01$,

$\mathrm{m}=\mu=0.005, \tau_{0}=0.05, \mathrm{~A}=$ 4 in $-10 \leq x, t \leq 10$.

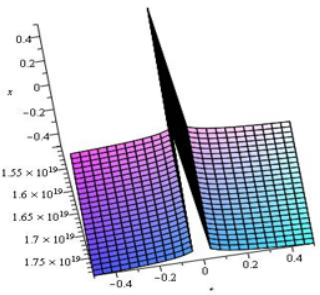

Figure 6: The anti-bell shape soliton of solution (3.2.15) for $\alpha=0.0001, \lambda=2, \mu=$ $0.01, \eta=0.04, \rho=0.1, \sigma=$ $2, y=0, \mathrm{~A}=3, B=1$ within $-0.5 \leq x, t \leq 0.5$. 


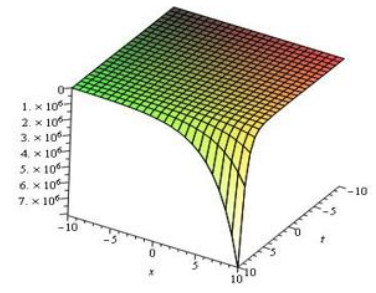

Figure 7: The shape of solution (3.2.17) for $\alpha=1, \lambda=0.2, \mu=$ $-0.3, \eta=5, \sigma=.002, \rho=$ $.0001, \mathrm{~A}=3, B=2, \mathrm{y}=0$ within $-10 \leq x, t \leq 10$.

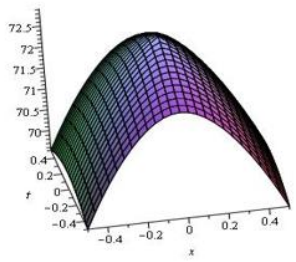

Figure 8: The plot of solution (3.2.18) for $\alpha=1, \lambda=0.2, \mu=$ $0.3, \eta=-0.4, \sigma=0.04, \rho=$

$0.2, \mathrm{~A}=3, B=2, \mathrm{y}=0, \tau_{0}=$ $0.02, \tau_{1}=0.03$ within $\quad-0.5 \leq$ $x, t \leq 0.5$.

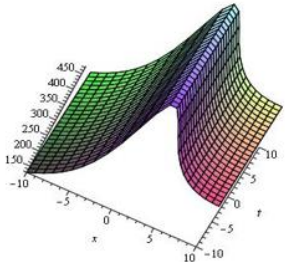

Figure 9: Shape of (3.2.19) for $\alpha=1, \lambda=0.02, \quad \mathrm{~A}=3, \quad \mu=$ -0.03 ,

$B=2, \eta=0.4, \sigma=5, \rho=0.2$,

$\tau_{1}=0.003, \mathrm{y}=0, \tau_{0}=0.002$ ， $-10 \leq x, t \leq 10$.

\section{Conclusions}

We aim to construct exact analytic solutions to the space-time fractional nonlinear Schrodinger equation and the (2+1)-dimensional time-fractional nonlinear Schrodinger equation by making use of the recently established rational $\left(D_{\xi}^{\alpha} G / G^{2}\right)$-expansion method. Consequently, three type's soliton solutions such as hyperbolic function, trigonometric function and rational function have been gained which are different from those of literature. The obtained solutions are outlined graphically to depict nonlinear complex physical phenomena arise in applied mathematics, mathematical physics and engineering. The performance of the employed method is claimed to be used for further research relating to the nonlinear models.

\section{REFERENCES}

Abdel-Salam, E., Yousif, E., El-Aasser, M. (2016). Analytical solution of the space-time fractional nonlinear Schrodinger equation. Rep. Math. Phys., 77, 19-34.

Alam, M. \& Li, X. (2019). Exact traveling wave solutions to higher order nonlinear equations. J. Ocean Eng. Sci., 4, 276-288.

Alam, M. (2015). Exact solutions to the foam drainage equation by using the new generalized $\left(G^{\prime} / G\right.$ expansion method. Res. Phys., 5, 168-177.

Alam, M., Akbar, M., Hoque, M. (2014). Exact traveling wave solutions of the (3+1)-dimensional $\mathrm{mKdV}-\mathrm{ZK}$ equation and the (1+1)-dimensional compound $\mathrm{KdVb}$ equation using new approach of the generalized $\left(G^{\prime} / G\right)$-expansion method. Pramana J. Phys., 83, 317-329.

Bazyar, M. H. \& Song, C. (2017). Analysis of transient wave scattering and its applications to site response analysis using the scaled boundary finite-element method. Soil Dyn. Earth quake Engg., 98, 191-205.

Bekir, A., Guner, O. A. (2013). Exact solutions of nonlinear fractional differential equations by $\left(G^{\prime} / G\right)$ expansion method. Chin. Phys. B, 103, 404-9.

Bhrawy, A. H., Ahmed, E. A., \& Baleanu, D. (2014a). The modified simple equation method for nonlinear fractional differential equation. Proc. Romanian Acad. A, 15, 322.

Bhrawy, A. H., Al-Zahrani, A. A., Alhamed, Y. A., Baleanu, D. (2014b).The modified simple equation method for nonlinear fractional differential equation. Rom. J. Phys. 59, 646.

Biswas, A., Ebadi, G., Triki, H., Yildirim, A., Yousefzadeh, N. (2013a). Topological soliton and other exact solutions to KdV-Caudrey-Dodd-Gibbon equation. Results Math, 63, 687-703. 
Biswas, A., Kumar, S., Krishnan, E. V., Ahmed, B., Strong, A., Johnson, S., Yildirim, A. (2013b). Topological solitons and other solutions to potential Korteweg-de-Vries equation. Rom. Rep. Phys., 65, 112537.

Doha, E. H., Baleanu, D., Bhrawy, A. H., Hafez, R. M. (2014). The modified simple equation method for nonlinear fractional differential equation. Proc. Romanian Acad. A. 15, 130.

Eslami, M., Vajargah, F., Mirzazadeh, M., \& Biswas, A. (2004). Application of first integral method to fractional partial differential equations. Indian J. Phys., 88, 177-184.

Ghanbari, B., Baleanu, D., Qurashi, M. A. (2019). New exact solutions of the generalized Benjamin Bonamahony equation. Symmerty 11, 20.

Golmankhaneh, A. K., Baleanu, D. (2011). Homotopy perturbation method for solving a system of Schrodinger-Korteweg-de Vries equation. Romanian Rep. Phys., 63, 609-623.

Guo, P. (2019). The Adomian decomposition method for a type of fractional differential equations. J. Apple. Math. Phys., 7, 2459-2466.

Islam, M. T. \& Akter, M. A. (2021a). Distinct solutions of nonlinear space-time fractional evolution equations appearing in mathematical physics via a new technique. Partial Diff. Eq. Appl. Math., 3.

Islam, M. T. \& Akter, M. A. (2021b). Exact analytic wave solutions to some nonlinear fractional differential equations for the shallow water wave arise in physics and engineering. J. Research Engg. Appl. Sci., 6(1), 11-18.

Jannah, M., Islam, M. T., Akter, M. A. (2021). Explicit travelling wave solutions to nonlinear partial differential equations arise in mathematical physics and engineering. J. Eng. Advan., 2, 58-63.

Kaplan, M., Unsal, O., Bekir. A. (2016). Exact solutions of nonlinear Schrodinger equation by using symbolic computation. Math. Meth. Appl. Sci., 39.

Karaagac, B. (2019). New exact solutions for some fractional order differential equations via improved sub-equation method. Discrete Contin. Dyn. Syst., 12, 447-454.

Khalil, R., Horani, M. A., Yousef, A., Sababheh, M. A. M. (2014). A new definition of fractional derivative. J. Comput. Appl. Math., 264, 65-70.

Kudryashov, N. A. (2012). One method for finding exact solutions of nonlinear differential equations. Commun. Nonlin. Sci. Numer. Simul., 17, 2248-53.

Laskin, N. (2002) Fractional Schrodinger equation. Phys. Rev. E., 66, 056108.

Li, C., Guo, Q., Zhao, M. (2019). On the solutions of (2+1)-dimensional time-fractional Schrodinger equation. Appl. Math. Lett., 94, 238-243.

Lu, D., Seadawy, A., Arshad, M. (2017). Application of extend simple equation method on unstable Schrodinger equations. Optic-Int. J. Light Elect. Opt., 140, 136-144.

Mohyud-Din, S. T. (2012). A meshless numerical solution of the family of generalized fifth-order Korteweg-de Vries equations. Int. J. Numer. Meth. Heat Fluid Flow, 2, 641-658.

Nuruddeen, R. I. \& Nass, A. M. (2017). Exact solutions of wave-type equations by the aboodh decomposition method. Stochastic Model Appl., 21, 23-30.

Nuruddeen, R. I. (2017). Elzaki decomposition method and its applications in solving linear and nonlinear Schrodinger equations. Sohag J. Math., 4, 1-5.

Omar, A. A. (2019a). Fitted fractional reproducing kernel algorithm for the numerical solutions of abcfractional Volterra integro-differential equations. Chaos Solit. Fract., 126, 394-402.

Omar, A. A. (2019b). Modulation of reproducing kernel hilbert space method for numerical solutions of Riccati and Bernoulli equations in the Atangana-Baleanu fractional sense. Chaos Solit. Fract., 125, 163-170.

Rizvi, S. T. R., Ali, K., Bashir, S., Younis, M., Ashraf, R., Ahmad, M. O. (2017). Exact solution of (2+1)dimensional fractional Schrodinger equation. Superlattices Microstruct., 107, 234-239. 
Saha, R. S. (2016). New analytical exact solutions of time fractional KdV-KZK equation by Kudryashov methods. Chin. Phys. B, 25, 040204.

Saxena, R., \& Kalla, S. (2010). Solution of space-time fractional Schrodinger equation occurring in quantum mechanics. Fract. Calc. Appl. Anal., 13, 177-190.

Shah, R., Khan, H., Arif, M., Kumam, P. (2019). Application of Laplace Adomian decomposition method for the analytical solution of third-order dispersive fractional partial differential equations. Entropy, 21, 335.

Sheikholeslami, M. (2017). Cuo-water nanofluid free convection in a porous cavity considering Darcy law. Eur. Phys. J. Plus, 132.

Younis, M., Rehman, H., Rizvi, S., \& Mahmood, S. (2017). Dark and singular optical solitons perturbation with fractional temporal evolution. Superlattices Microstruct, 104, 525-531.

Zayed, E. M. \& Alurrfi, K. A. (2016). The $\left(G^{\prime} / G, 1 / G\right)$-expansion method and its applications to two nonlinear Schrodinger equations describing the propagation of femtosecond pulses in nonlinear optical fibers. Optik-International Journal for Light and Electron Optics, 127, 1581-1589.

Zayed, E. M. E. \& Abdelaziz, M. A. M. (2012). The two-variable $G^{\prime} / G, 1 / G$-expansion method for solving the nonlinear KdV-mKdV equation. Math. Prob. Engg., 14, 725061.

Zayed, E. M., Shahoot, A. M., Alurrfi, K.A. (2018). The $\left(G^{\prime} / G, 1 / G\right)$-expansion method and its applications for constructing many new exact solutions of the higher-order nonlinear Schrodinger equation and the quantum Zakharov Kuznetsov equation. Optical and Quantum Electronics, 50, 96.

$--0--$ 